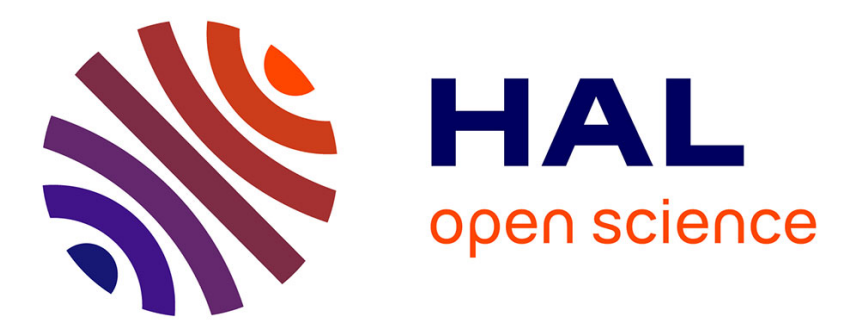

\title{
Modified policy iteration algorithms are not strongly polynomial for discounted dynamic programming
}

\author{
Eugene A. Feinberg, Jefferson Huang, Bruno Scherrer
}

\section{To cite this version:}

Eugene A. Feinberg, Jefferson Huang, Bruno Scherrer. Modified policy iteration algorithms are not strongly polynomial for discounted dynamic programming. Operations Research Letters, 2014, 42, pp.429 - 431. 10.1016/j.orl.2014.07.006 . hal-01091370

\section{HAL Id: hal-01091370 \\ https://hal.inria.fr/hal-01091370}

Submitted on 5 Dec 2014

HAL is a multi-disciplinary open access archive for the deposit and dissemination of scientific research documents, whether they are published or not. The documents may come from teaching and research institutions in France or abroad, or from public or private research centers.
L'archive ouverte pluridisciplinaire HAL, est destinée au dépôt et à la diffusion de documents scientifiques de niveau recherche, publiés ou non, émanant des établissements d'enseignement et de recherche français ou étrangers, des laboratoires publics ou privés. 


\title{
Modified policy iteration algorithms are not strongly polynomial for discounted dynamic programming
}

\author{
Eugene A. Feinberg ${ }^{\mathrm{a}, *}$, Jefferson Huang ${ }^{\mathrm{a}}$, Bruno Scherrer ${ }^{\mathrm{b}, \mathrm{c}}$ \\ ${ }^{a}$ Department of Applied Mathematics and Statistics, Stony Brook University, Stony Brook, NY 11794-3600, USA \\ ${ }^{b}$ Inria, Villers-lès-Nancy, F-54600, France \\ ${ }^{c}$ Université de Lorraine, LORIA, UMR 7503, Vandoeuvre-lès-Nancy, F-54506, France
}

\begin{abstract}
This note shows that the number of arithmetic operations required by any member of a broad class of optimistic policy iteration algorithms to solve a deterministic discounted dynamic programming problem with three states and four actions may grow arbitrarily. Therefore any such algorithm is not strongly polynomial. In particular, the modified policy iteration and $\lambda$-policy iteration algorithms are not strongly polynomial.
\end{abstract}

Keywords: Markov decision process, Modified policy iteration, Strongly polynomial, Policy, Algorithm

\section{Introduction}

Value iteration (VI), policy iteration (PI), and linear programming algorithms are three major methods for computing optimal policies for Markov decision Processes (MDPs) with expected total discounted rewards ([8], [11, Ch. 6]), also known under the name of discounted dynamic programming. As is well-known, PI can be viewed as an implementation of the simplex method applied to one of the two major linear programs used to solve MDPs; see e.g. [8], [11, §6.9]. Using these linear programs, Ye [16] proved that both Howard's [7] PI and the simplex method with Dantzig's pivoting rule are strongly polynomial when the discount factor is fixed; in other words, taking the discount factor to be a constant, the number of arithmetic operations needed by these two algorithms to return an optimal policy is bounded above by a polynomial function of the number of state-action pairs $m$. Post \& Ye [10] subsequently showed that, if the MDP is deterministic, then the simplex method with Dantzig's rule is strongly polynomial regardless of the discount factor. In contrast, Feinberg \& Huang [5] used a deterministic MDP to demonstrate that VI is not strongly polynomial even when the discount factor is fixed. As was proved by Tseng [15], the VI algorithm is weakly polynomial, that is, the number

\footnotetext{
${ }^{*}$ Corresponding author.

Email address: eugene. feinberg@stonybrook. edu (Eugene A. Feinberg)
}

Preprint submitted to Operations Research Letters of required arithmetic operations can be bounded above by a polynomial in $m$ and the total bit-size of the input data.

Each iteration of PI involves the solution of a system of linear equations, which may be time consuming if the number of states is large. Several methods have been proposed to deal with this issue by combining the advantages of PI and VI. One approach is modified policy iteration (MPI), where the exact solutions are replaced with estimates obtained via finite numbers of successive approximations; see Puterman \& Shin [12]. Another approach is $\lambda$-policy iteration ( $\lambda \mathrm{PI})$, also called temporal difference-based policy iteration; see Bertsekas \& Tsitsiklis [2, §2.3.1]. Both of these algorithms include VI and PI as special cases. In studying performance bounds for approximate versions of $\lambda$ PI, Thiéry \& Scherrer [14] considered a generalization of both MPI and $\lambda$ PI, which they refer to as optimistic policy iteration (OPI). In this note, we use a variant of Feinberg \& Huang's [5] example to show that a generalization of OPI, which we call generalized optimistic policy iteration $(G-O P I)$, is not strongly polynomial (Theorem 1). In particular, our result implies that VI, MPI, $\lambda$ PI, and OPI are also not strongly polynomial (Corollary 2).

We remark that the results in Ye [16] have led to further developments. For instance, Hansen Miltersen \& Zwick [6] improved the iteration bound for Howard's PI given in [16] by a factor of the number of states $n$, and showed that it also applies to the strategy iteration algorithm for two-player turn-based zero-sum stochas- 
tic games. Scherrer [13] improved both the estimate in [6] for Howard's PI and the estimate in [16] for the simplex method by a factor of $\ln (n)$, showing that if the discount factor is fixed then Howard's PI needs at most a linear number of iterations in $m$ and the simplex method with Dantzig's rule needs at most a linear number of iterations in $m n$. The results and analysis in Ye [16] have also been applied in both more general and different contexts. Kitahara \& Mizuno [9] used the analysis in [16] to obtain a sufficient condition for the simplex method to be strongly polynomial for linear programs in general. In addition, Ye [16, $\S 5]$ notes that the analysis of discounted MDPs can be extended to transient MDPs; Denardo [3] showed that with some modifications, the analysis given in [16, §5] can yield a bound improved by a factor of 2 for such MDPs. Finally, the results in [16] are relevant for certain MDPs under the average-reward criterion; see Feinberg \& Huang [4] and Akian \& Gaubert [1].

\section{Generalized optimistic policy iteration}

In Section 2.1 we describe the discounted-reward criterion. In Section 2.2, we formulate the G-OPI algorithm and state our results, namely Theorem 1 and Corollary 2, which are proved in Section 3 .

\subsection{Discounted-reward criterion}

Consider a discrete-time MDP with finite state set $\mathbb{X}$, finite nonempty sets of actions $A(x)$ available at each $x \in \mathbb{X}$, transition probabilities $p(y \mid x, a)$ for each $x, y \in$ $\mathbb{X}$ and $a \in A(x)$, and one-step rewards $r(x, a)$ for each $x \in \mathbb{X}$ and $a \in A(x)$. Let $m:=\sum_{x \in \mathbb{X}}|A(x)|$ denote the total number of state-action pairs.

Here we are interested in maximizing expected infinite-horizon discounted rewards. In particular, a policy is a mapping $\phi: \mathbb{X} \rightarrow \bigcup_{x \in \mathbb{X}} A(x)$ such that $\phi(x) \in$ $A(x)$ for each $x \in \mathbb{X}$. One may consider more general policies, but for infinite-horizon discounted MDPs with finite state and action sets it is sufficient to consider only policies of this form; see e.g. [11, p. 154]. Let $F$ denote the set of all policies. Also, given an initial state $x \in \mathbb{X}$, let $\mathbb{P}_{x}^{\phi}$ denote the probability distribution on the set of possible histories $x_{0} a_{0} x_{1} a_{1} \ldots$ of the process under the policy $\phi$ with $x_{0}=x$, and let $\mathbb{E}_{x}^{\phi}$ be the expectation operator associated with $\mathbb{P}_{x}^{\phi}$. Then, letting $\beta \in(0,1)$ denote the discount factor, the expected total discounted reward earned when the policy $\phi$ is used starting from state $x \in \mathbb{X}$ is

$$
v_{\beta}(x, \phi):=\mathbb{E}_{x}^{\phi} \sum_{t=0}^{\infty} \beta^{t} r\left(x_{t}, a_{t}\right) .
$$

The goal is to find an optimal policy, i.e. a policy $\phi^{*}$ such that $v_{\beta}\left(x, \phi^{*}\right)=\sup _{\phi \in F} v_{\beta}(x, \phi)$ for all $x \in \mathbb{X}$. It is well-known that if $\mathbb{X}$ and $\bigcup_{x \in \mathbb{X}} A(x)$ are finite, then an optimal policy exists; see e.g. [11, p. 154]. To describe the G-OPI algorithm, it will be convenient to define the operators $T$ and $T_{\phi}, \phi \in F$, on functions $v: \mathbb{X} \rightarrow \mathbb{R}$ for each $x \in \mathbb{X}$ by

$$
T v(x):=\max _{a \in A(x)}\left\{r(x, a)+\beta \sum_{y \in \mathbb{X}} p(y \mid x, a) v(y)\right\}
$$

and

$$
T_{\phi} v(x):=r(x, \phi(x))+\beta \sum_{y \in \mathbb{X}} p(y \mid x, \phi(x)) v(y),
$$

where for $n=1,2, \ldots, T_{\phi}^{0} v(x):=v(x)$ and $T_{\phi}^{n} v(x):=T_{\phi}\left(T_{\phi}^{n-1} v\right)(x)$.

\subsection{The algorithm}

Algorithm 1. (G-OPI) Let $\mathbb{N}$ denote the set of positive integers, $\overline{\mathbb{N}}:=\mathbb{N} \cup\{+\infty\}$, and let $\left\{N_{j}\right\}_{j=1}^{\infty}$ be an $\overline{\mathbb{N}}$ valued stochastic sequence with associated probability measure $P$ and expectation operator $E$. Then given $V_{0}$ : $\mathbb{X} \rightarrow \mathbb{R}$, set $j=1$ and choose any policy $\phi^{j}$ satisfying

$$
T_{\phi^{j}} V_{j-1}(x)=T V_{j-1}(x) \text { for each } x \in \mathbb{X} .
$$

If $V_{j-1}(x)=T V_{j-1}(x)$ for all $x \in \mathbb{X}$, then $\phi^{j}$ is an optimal policy; otherwise, set

$$
V_{j}(x)=E\left[T_{\phi^{j}}^{N_{j}} V_{j-1}(x)\right] \quad \text { for each } x \in \mathbb{X},
$$

increase $j$ by 1 , and repeat starting from (1).

In the sequel, we assume that a strongly polynomial algorithm exists for evaluating the expectation in (2) for each $j \in \mathbb{N}$; otherwise, it trivially follows that the GOPI algorithm is not strongly polynomial. The following statement, which is proved in Section 3 , is the main result of this note.

\section{Theorem 1. If}

$$
P\left\{N_{j}<+\infty\right\}>0 \text { for each } j \in \mathbb{N},
$$

then the number of iterations needed by the generalized optimistic policy iteration algorithm to return the optimal policy may grow arbitrarily quickly as the number of state-action pairs $m$ increases, which implies that the algorithm is not strongly polynomial.

The generalized optimistic policy iteration algorithm includes VI, MPI, $\lambda$ PI, OPI, and Howard's PI as special cases. In fact, we show in Section 3 that Theorem 1 implies 
Corollary 2. The value iteration, modified policy iteration, $\lambda$-policy iteration, and optimistic policy iteration algorithms are not strongly polynomial.

Note that Theorem 1 does not apply to Howard's PI, under which $P\left\{N_{j}=+\infty\right\}=1$ for each $j \in \mathbb{N}$, and which is strongly polynomial according to Ye [16].

\section{Proofs}

To prove Theorem 1 we shall consider the following example.

Example 1. Let the state set be $\mathbb{X}=\{1,2,3\}$, and given a positive integer $k$, let $A(1)=\{0,1\}, A(2)=$ $\{0\}$, and $A(3)=\{0\}$ be the sets of actions available at states 1,2 , and 3 , respectively; hence the number of state-action pairs $m=4$. The transition probabilities are $p(2 \mid 1,1)=p(3 \mid 1,0)=p(2 \mid 2,0)=p(3 \mid 3,0)=1$. Finally, the one-step rewards are $r(1,0)=r(2,0)=0$, $r(3,0)=1$, and $r(1,1)=R<\beta /(1-\beta)$. Figure 1 illustrates this MDP.

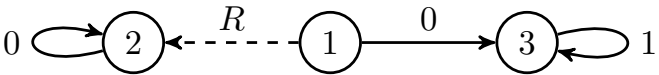

Figure 1: The solid arcs correspond to transitions associated with action 0 , and the dashed arc corresponds to action 1 . The number next to each arc is the one-step reward that taking the corresponding action earns.

For this MDP, each policy is characterized by the action selected at state 1 . If action 1 is selected, then the total discounted reward starting from state 1 is $R<$ $\beta /(1-\beta)$; if action 0 is selected, the corresponding total discounted reward is $\beta /(1-\beta)$. Hence action 0 is the optimal action at state 1 .

Proof of Theorem $[$ Apply the G-OPI algorithm to the MDP in Example 11 with $V_{0}(1)=V_{0}(2)=V_{0}(3)=0$. From (2),

$$
\begin{aligned}
V_{1}(3) & =E\left[1+\beta+\cdots+\beta^{N_{1}-1}+\beta^{N_{1}} \cdot 0\right] \\
& =E\left[1-\beta^{N_{1}}\right] /(1-\beta) \\
& =\frac{1}{1-\beta}\left(1-E\left[\beta^{N_{1}}\right]\right)
\end{aligned}
$$

and

$$
\begin{aligned}
V_{2}(3) & =E\left[1+\beta+\cdots+\beta^{N_{2}-1}+\beta^{N_{2}} V_{1}(3)\right] \\
& =\frac{1}{1-\beta}\left(1-E\left[\beta^{N_{2}}\right]+E\left[\beta^{N_{2}}\left(1-E\left[\beta^{N_{1}}\right]\right)\right]\right) \\
& =\frac{1}{1-\beta}\left(1-E\left[\beta^{N_{2}}\right] E\left[\beta^{N_{1}}\right]\right) .
\end{aligned}
$$

Hence by induction,

$$
\begin{aligned}
V_{j}(3) & =E\left[1+\beta+\cdots+\beta^{N_{j}-1}+\beta^{N_{j}} V_{j-1}(3)\right] \\
& =\frac{1}{1-\beta}\left(1-\prod_{\ell=1}^{j} E\left[\beta^{N_{\ell}}\right]\right) \quad \text { for each } j \in \mathbb{N} .
\end{aligned}
$$

This means the optimal action 0 at state 1 will be selected on iteration $j^{*}$ only if

$$
\beta V_{j^{*}-1}(3)=\frac{\beta}{1-\beta}\left(1-\prod_{\ell=1}^{j^{*}-1} E\left[\beta^{N_{\ell}}\right]\right) \geq R .
$$

Suppose $P\left\{N_{j}<+\infty\right\}=\sum_{n=1}^{\infty} P\left\{N_{j}=n\right\}>0$ for each $j \in \mathbb{N}$. Then $P\left\{N_{j}=n_{0}\right\}>0$ for some $n_{0} \in \mathbb{N}$; since $\beta>0$ and $\beta^{+\infty}=0$ this implies

$$
\begin{aligned}
E\left[\beta^{N_{j}}\right] & =\sum_{n=1}^{\infty} \beta^{n} P\left\{N_{j}=n\right\} \\
& \geq \beta^{n_{0}} P\left\{N_{j}=n_{0}\right\}>0 \quad \text { for each } j \in \mathbb{N} .
\end{aligned}
$$

It follows that

$$
\begin{aligned}
\beta V_{j}(3) & =\frac{\beta}{1-\beta} \cdot\left(1-\prod_{\ell=1}^{j} E\left[\beta^{N_{\ell}}\right]\right) \\
& <\frac{\beta}{1-\beta} \quad \text { for each } j \in \mathbb{N} .
\end{aligned}
$$

Hence, for any $k<\infty, R$ may be chosen such that for all $j \leq k$,

$$
\frac{\beta}{1-\beta}>R>\beta V_{j}(3) \text {. }
$$

In other words, the number of iterations before the algorithm switches to the optimal action 0 can be arbitrarily large.

Proof of Corollary 2 The VI, MPI, $\lambda$ PI, and OPI algorithms differ from G-OPI only in how $V_{j}$ is computed in (2). For VI, (2) is replaced with

$$
V_{j}(x)=T_{\phi^{j}} V_{j-1}(x) \quad \text { for each } x \in \mathbb{X},
$$

so $P\left\{N_{j}<+\infty\right\}=P\left\{N_{j}=1\right\}=1$ for all $j \in \mathbb{N}$. For MPI, each $N_{j}$ is simply a constant $n_{j} \in \mathbb{N}$, so (2) can be written as

$$
V_{j}(x)=T_{\phi^{j}}^{n_{j}} V_{j-1}(x) \quad \text { for each } x \in \mathbb{X}
$$

and $P\left\{N_{j}<+\infty\right\}=P\left\{N_{j}=n_{j}\right\}=1$ for each $j \in \mathbb{N}$. For $\lambda$ PI, each $N_{j}$ is an independent geometric random variable, i.e. for $j \in \mathbb{N}$

$$
P\left\{N_{j}=n\right\}=\left(1-\lambda_{j}\right) \lambda_{j}^{n-1}, \quad \lambda_{j} \in[0,1), n \in \mathbb{N},
$$


implying that $P\left\{N_{j}<+\infty\right\}=1$ for each $j \in \mathbb{N}$. Finally, under the OPI algorithm the distribution of each $N_{j}$ is defined by a sequence $\left\{\lambda_{n}^{j}\right\}_{n=1}^{\infty}$ of nonnegative numbers satisfying $\sum_{n=1}^{\infty} \lambda_{n}^{j}=1$, where

$$
P\left\{N_{j}=n\right\}=\lambda_{n}^{j}, \quad n \in \mathbb{N} ;
$$

hence $P\left\{N_{j}<+\infty\right\}=\sum_{n=1}^{\infty} \lambda_{n}^{j}=1$ for all $j \in \mathbb{N}$. Hence each of these algorithms is an instance of G-OPI where $P\left\{N_{j}<+\infty\right\}>0$ for each $j \in \mathbb{N}$.

Acknowledgement. The research of the first two authors was partially supported by NSF grant CMMI1335296.

\section{References}

[1] M. Akian and S. Gaubert. Policy iteration for perfect information stochastic mean payoff games with bounded first return times is strongly polynomial. Preprint, 2013. http://arxiv.org/abs/1310.4953v1

[2] D. P. Bertsekas and J. N. Tsitsiklis. Neuro-Dynamic Programming. Athena Scientific, Belmont, MA, 1996.

[3] E. V. Denardo. Nearly strongly polynomial algorithms for transient Markov decision problems. Unpublished Manuscript, 2014.

[4] E. A. Feinberg and J. Huang. Strong polynomiality of policy iterations for average-cost MDPs modeling replacement and maintenance problems. Operations Research Letters, 41(3):249-251, 2013.

[5] E. A. Feinberg and J. Huang. The value iteration algorithm is not strongly polynomial for discounted dynamic programming. Operations Research Letters, 42(2):130-131, 2014.

[6] T. D. Hansen, P. B. Miltersen, and U. Zwick. Strategy iteration is strongly polynomial for 2-player turn-based stochastic games with a constant discount factor. Journal of the ACM, 60(1):Article 1, 16 pages, 2013.

[7] R. A. Howard. Dynamic Programming and Markov Processes. The MIT Press, Cambridge, MA, 1960.

[8] L. C. M. Kallenberg. Finite state and action MDPs. In E. A. Feinberg and A. Schwartz, editors, Handbook of Markov Decision Processes, pages 21-87. Kluwer, Boston, 2002.

[9] T. Kitahara and S. Mizuno. A bound for the number of different basic solutions generated by the simplex method. Mathematical Programming Ser. A, 137:579-586, 2013.

[10] I. Post and Y. Ye. The simplex method is strongly polynomial for deterministic Markov decision processes. to appear in Mathematics of Operations Research, 2014.

[11] M. L. Puterman. Markov Decision Processes: Discrete Stochastic Dynamic Programming. John Wiley \& Sons, Inc., New York, 1994.

[12] M. L. Puterman and M. C. Shin. Modified policy iteration algorithms for discounted Markov decision problems. Management Science, 24(11):1127-1137, 1978.

[13] B. Scherrer. Improved and generalized upper bounds on the complexity of policy iteration. In C.J.C. Burges, L. Bottou, M. Welling, Z. Ghahramani, and K.Q. Weinberger, editors, Advances in Neural Information Processing Systems 26, pages 386-394. NIPS Foundation, Inc., 2013.

[14] Christophe Thiery and Bruno Scherrer. Least-squares policy iteration: Bias-variance trade-off in control problems. In Johannes Fürnkranz and Thorsten Joachims, editors, Proceedings of the 27th International Conference on Machine Learning
(ICML-10), pages 1071-1078, Haifa, Israel, June 2010. Omnipress.

[15] P. Tseng. Solving h-horizon, stationary Markov decision problems in time proportional to $\log (\mathrm{h})$. Operations Research Letters, 9(5):287-297, 1990.

[16] Y. Ye. The simplex and policy-iteration methods are strongly polynomial for the Markov decision problem with a fixed discount rate. Mathematics of Operations Research, 36(4):593603, 2011. 